\title{
How well can skin marker analysis detect the kinematics of a total ankle arthroplasty? - a comparison to videofluoroscopy
}

\author{
Renate List $^{1 *}$, Hans Gerber ${ }^{1}$, Mauro Foresti', Silvio Lorenzetti ${ }^{1}$, Pascal Rippstein ${ }^{2}$, Edgar Stüssi ${ }^{1}$ \\ From 3rd Congress of the International Foot and Ankle Biomechanics Community \\ Sydney, Australia. 11-13 April 2012
}

\section{Background}

Previous in vivo studies on total ankle arthroplasty (TAA) kinematics were mainly performed using skin marker analysis, which has the drawback of skin movement artefacts [1]. A further limitation is the inaccessibility of the talus for attaching markers, thus the impossibility to distinguish tibiotalar from subtalar motion. So far it is not known how well skin marker analysis detects the kinematics of the TAA.

\section{Materials and methods}

The kinematics of 11 TAA participants were simultaneously analysed by skin marker and videofluoroscopic assessment during level gait (gt), walking up- (uph) and downhill (dnh). The fluoroscopic data analysis included a $2 \mathrm{D} / 3 \mathrm{D}$ registration (error $<0.2^{\circ}$ in-plane, $<1.3^{\circ}$ out-ofplane) [2]. The markerset consisted of 4 rearfoot and 6 shank markers [3]. For both approaches joint rotations were described along the axes of the marker based joint coordinate system. As a descriptor of differentiation the maximal and the root mean square differences (max diff, RMS diff) between skin marker and fluoroscopic joint rotations were calculated over the whole stance phase. Besides, maximal ranges of motion (ROM) were compared using a paired t-test.

\section{Results}

Skin marker analysis significantly overestimated sagittal plane ROM of the TAA for $5(\mathrm{gt}), 6(\mathrm{uph})$ and $6(\mathrm{dnh})$ and underestimated for 1 (uph) and 2 (dnh) subjects.

Table 1 RMS diff and max diff over the whole stance phase and ROM assessed by videofluoroscopy (fluoro) and skin marker analysis (skin). Mean and SD over all 11 subjects of sagittal (sag), frontal (front) and transverse (trans) plane rotations, * statistically significant difference between fluoro and and skin $(p<0.05)$.

\begin{tabular}{|c|c|c|c|c|c|c|c|c|c|c|c|c|c|c|c|c|c|c|c|}
\hline & \multicolumn{6}{|c|}{ Level gait } & \multicolumn{6}{|c|}{ Uphill } & \multicolumn{6}{|c|}{ Downhill } \\
\hline & & \multicolumn{2}{|c|}{ Sag } & \multicolumn{2}{|c|}{ Front } & \multicolumn{2}{|c|}{ Trans } & \multicolumn{2}{|c|}{ Sag } & \multicolumn{2}{|c|}{ Front } & \multicolumn{2}{|c|}{ Trans } & \multicolumn{2}{|c|}{ Sag } & \multicolumn{2}{|c|}{ Front } & \multicolumn{2}{|c|}{ Trans } \\
\hline $\begin{array}{l}\text { RMS } \\
\text { diff } \\
{\left[{ }^{\circ}\right]}\end{array}$ & $\begin{array}{l}\text { Mean } \\
\pm \text { SD }\end{array}$ & \multicolumn{2}{|c|}{$\begin{array}{c}1.5 \\
\pm 0.7\end{array}$} & \multicolumn{2}{|c|}{$\begin{array}{c}2.0 \\
\pm 0.9\end{array}$} & \multicolumn{2}{|c|}{$\begin{array}{c}2.9 \\
\pm 1.0\end{array}$} & \multicolumn{2}{|c|}{$\begin{array}{c}1.5 \\
\pm 0.6\end{array}$} & \multicolumn{2}{|c|}{$\begin{array}{c}2.4 \\
\pm 1.4\end{array}$} & \multicolumn{2}{|c|}{$\begin{array}{c}2.8 \\
\pm 1.2\end{array}$} & \multicolumn{2}{|c|}{$\begin{array}{c}1.7 \\
\pm 0.6\end{array}$} & \multicolumn{2}{|c|}{$\begin{array}{c}2.0 \\
\pm 0.9\end{array}$} & \multicolumn{2}{|c|}{$\begin{array}{c}3.0 \\
\pm 1.2\end{array}$} \\
\hline \multirow{2}{*}{$\begin{array}{l}\text { Max } \\
\text { diff } \\
{\left[{ }^{\circ}\right]}\end{array}$} & $\begin{array}{l}\text { Mean } \\
\pm \text { SD }\end{array}$ & \multicolumn{2}{|c|}{$\begin{array}{c}3.9 \\
\pm 1.7\end{array}$} & \multicolumn{2}{|c|}{$\begin{array}{c}4.9 \\
\pm 2.5\end{array}$} & \multicolumn{2}{|c|}{$\begin{array}{c}6.8 \\
\pm 2.7\end{array}$} & \multicolumn{2}{|c|}{$\begin{array}{c}3.6 \\
\pm 1.8\end{array}$} & \multicolumn{2}{|c|}{$\begin{array}{c}5.1 \\
\pm 2.8\end{array}$} & \multicolumn{2}{|c|}{$\begin{array}{c}5.7 \\
\pm 2.2\end{array}$} & \multicolumn{2}{|c|}{$\begin{array}{c}4.3 \\
\pm 1.7\end{array}$} & \multicolumn{2}{|c|}{$\begin{array}{c}4.5 \\
\pm 1.9\end{array}$} & \multicolumn{2}{|c|}{$\begin{array}{c}5.7 \\
\pm 1.9\end{array}$} \\
\hline & & Fluoro & Skin & Fluoro & Skin & Fluoro & Skin & Fluoro & Skin & Fluoro & Skin & Fluoro & Skin & Fluoro & Skin & Fluoro & Skin & Fluoro & Skin \\
\hline $\begin{array}{l}\text { ROM } \\
\left.{ }^{\circ}{ }^{\circ}\right]\end{array}$ & $\begin{array}{l}\text { Mean } \\
\pm \text { SD }\end{array}$ & $\begin{array}{l}10.0^{*} \\
\pm 2.8\end{array}$ & $\begin{array}{c}11.6^{*} \\
\pm \\
2.9\end{array}$ & $\begin{array}{c}2.9^{*} \\
\pm 1.0\end{array}$ & $\begin{array}{c}5.8^{*} \\
\pm \\
2.1\end{array}$ & $\begin{array}{c}8.1^{*} \\
\pm 2.9\end{array}$ & $\begin{array}{c}6.5^{*} \\
\pm \\
2.7\end{array}$ & $\begin{array}{c}9.6^{*} \\
\pm 4.8\end{array}$ & $\begin{array}{c}11.5^{*} \\
\pm \\
3.3\end{array}$ & $\begin{array}{c}2.5^{*} \\
\pm 0.7\end{array}$ & $\begin{array}{c}5.6^{*} \\
\pm \\
2.7\end{array}$ & $\begin{array}{c}6.6 \\
\pm 2.3\end{array}$ & $\begin{array}{c}6.8 \\
\pm \\
3.2\end{array}$ & $\begin{array}{c}13.1 \\
\pm 3.7\end{array}$ & $\begin{array}{c}14.7 \\
\pm \\
2.7\end{array}$ & $\begin{array}{c}3.0^{*} \\
\pm 0.6\end{array}$ & $\begin{array}{c}5.4^{*} \\
\pm \\
2.1\end{array}$ & $\begin{array}{c}7.7^{*} \\
\pm 2.5\end{array}$ & $\begin{array}{c}5.7^{*} \\
\pm \\
2.3\end{array}$ \\
\hline
\end{tabular}

\footnotetext{
* Correspondence: rlist@ethz.ch

${ }^{1}$ Institute for Biomechanics, ETH Zurich, 8093 Zurich, Switzerland
}

Full list of author information is available at the end of the article 
Frontal plane ROM was significantly overestimated for 7 (gt), 8(uph) and $9(\mathrm{dnh})$ of the 11 subjects. Transverse plane ROM was for 2(uph) and 2(dnh) subjects significantly overestimated, and for 3(gt), 1(uph) and 7(dnh) subjects significantly underestimated by skin markers. For mean RMS diff, mean max diff and mean ROM see Table 1.

\section{Conclusions}

The differences between skin marker assessed rearfootshank and the fluoroscopic assessed isolated TAA motion were neither consistent between subjects, nor motion planes, nor conditions. For transverse and frontal plane rotations, the maximal differences were in the range of the maximal corresponding ROM. Discrepancies for the sagittal plane were smaller, but still for some subjects, ROM were significantly different.

\section{Author details}

IInstitute for Biomechanics, ETH Zurich, 8093 Zurich, Switzerland. ${ }^{2}$ Foot and Ankle Center, Schulthess Clinic, 8093 Zurich, Switzerland.

Published: 10 April 2012

\section{References}

1. Leardini A, Chiari L, Della Croce U, Cappozzo A: Human movement analysis using stereophoto-grammetry. Part 3 . Soft tissue artifact assessment and compensation. Gait Posture 2005, 21:212-225.

2. List R, Stacoff A, Foresti M, Gerber H, Stuessi E: An in vivo procedure to quantify 3D kinematics of ankle arthroplasties using videofluoroscopy. J Biomech 2008, 41:S321.

3. List R, Unternährer S, Ukelo T, Wolf P, Stacoff A: Erfassen der Vor- und Rückfussbewegungen im Gehen und Laufen. Sportmed Sporttraumatol 2008, 56:43-49.

doi:10.1186/1757-1146-5-S1-O35

Cite this article as: List et al:: How well can skin marker analysis detect the kinematics of a total ankle arthroplasty? - a comparison to videofluoroscopy. Journal of Foot and Ankle Research 2012 5(Suppl 1):035.

\section{Submit your next manuscript to BioMed Central} and take full advantage of:

- Convenient online submission

- Thorough peer review

- No space constraints or color figure charges

- Immediate publication on acceptance

- Inclusion in PubMed, CAS, Scopus and Google Scholar

- Research which is freely available for redistribution

Submit your manuscript at www.biomedcentral.com/submit
C Biomed Central 